Supplement of Earth Syst. Dynam., 11, 721-735, 2020

https://doi.org/10.5194/esd-11-721-2020-supplement

(C) Author(s) 2020. This work is distributed under

the Creative Commons Attribution 4.0 License.

(c) (1)
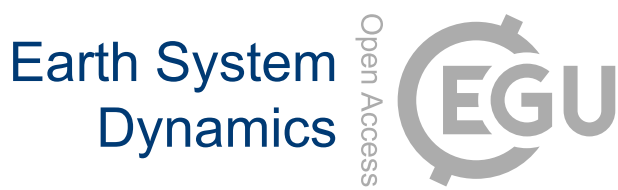

Supplement of

\title{
Relating climate sensitivity indices to projection uncertainty
}

\section{Benjamin Sanderson}

Correspondence to: Benjamin Sanderson (sanderson@cerfacs.fr)

The copyright of individual parts of the supplement might differ from the CC BY 4.0 License. 


\section{S1 Additional Figures}

Figure S1. Plots illustrating how different types of sensitivity metric are influenced by climatological drift. Each line describes the evolution of the model (with default parameters), where the control simulation is initialized 500 years in advance of the sensitivity experiment with a non-zero forcing ranging from -1 to $1 \mathrm{Wm}^{-2}$. (a) shows the global mean temperature time evolution of the abrupt4xCO2 simulations (blue) and the 1pctCO2 simulation (green), with box-whisker plots showing the range of biased values which are measured due to climate drift for A140, T140 and EffCS. (b) shows the trend lines used to compute the EffCS estimates from the simple model. Blue lines show an example model configuration response to an abrupt $4 \mathrm{xCO} 2$ perturbation in for the equilibrated case (dashed), and end members $\left(+/-1 \mathrm{Wm}^{-2}\right.$ imbalance). The red shaded area shows the range of fitted trend lines consistent with (a).

(a)

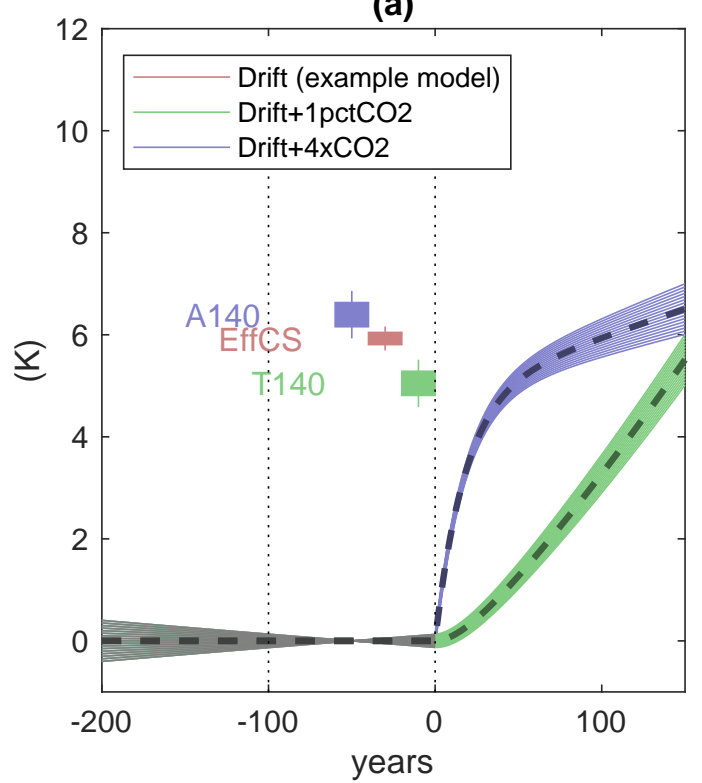

(b)

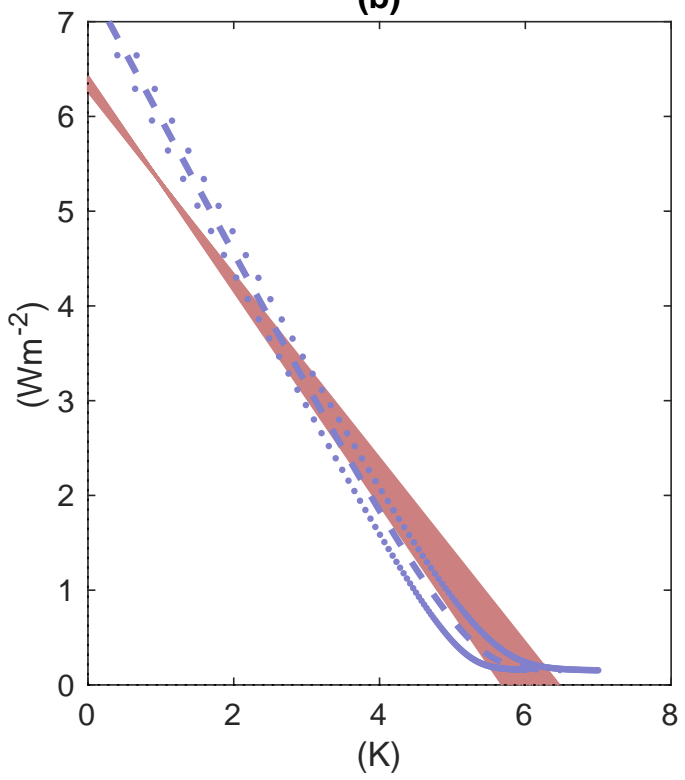


Figure S2. Scatterplots of 21st century warming (difference between 20 year means in 2081-2100 and 1981-2000) and a range of sensitivity metrics for CMIP5. TCR, T140 and EffCS are reported values from IPCC (2013), A140 is calculated as the year 131-150 average global mean temperature above the control level (taken as the last 100 years of the relevant control simulation). Columns represent different RCPs, rows represent different sensitivity metrics considered in the text. Each point represents a single model from the archive. Only results from the 1 st initial condition ensemble member are considered for each model (thus the plots are subject to initial condition variability).
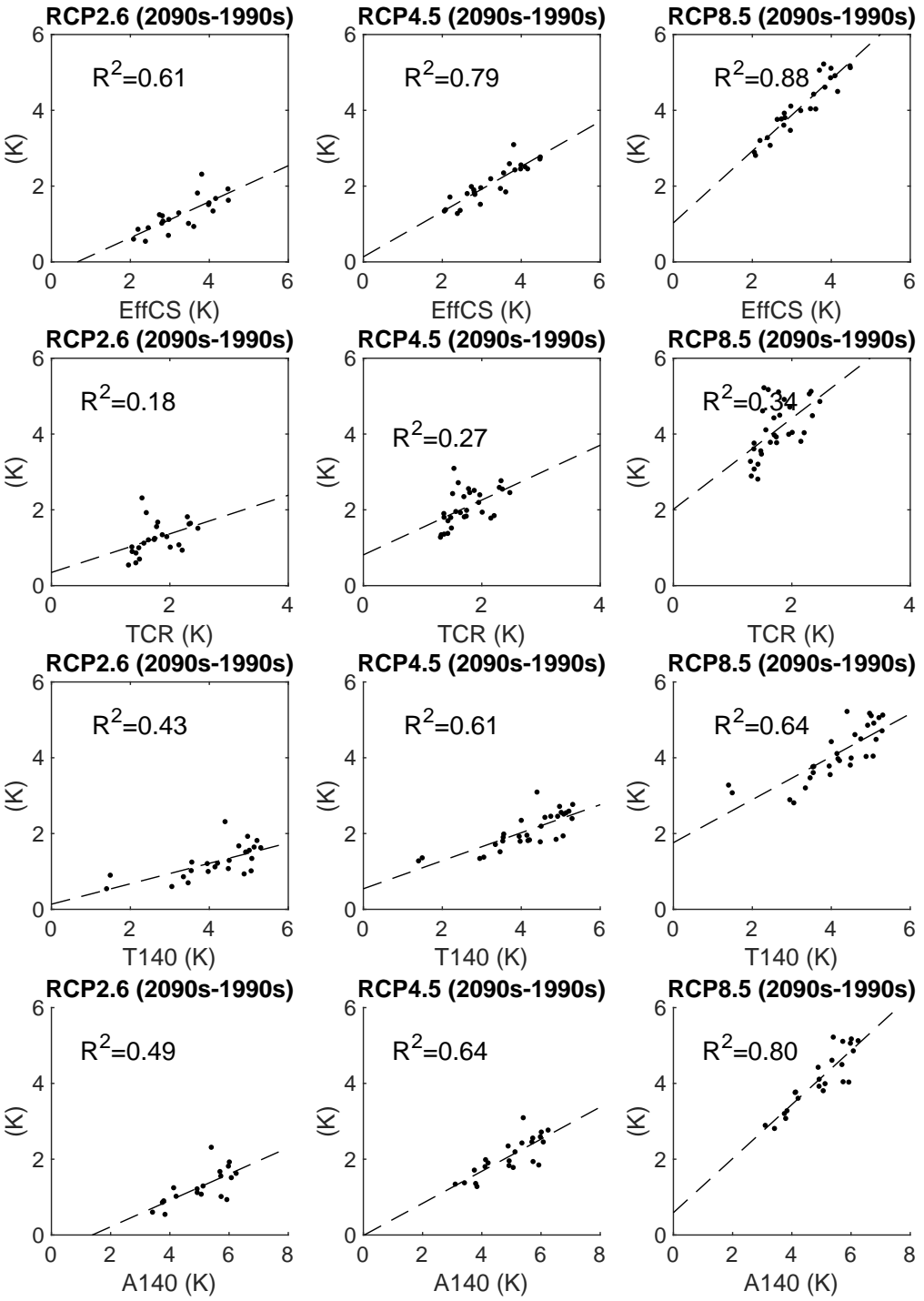
Figure S3. A demonstration of the simple model fitting strategy applied to historical simulations for a range of models in the CMIP5 archive. A pulse-response model is fitted treating each model's global mean temperature output in turn as truth for the period 1870-2019 (black line). 10th-90th percentiles of fitted temperature response for historical (grey area) and future projections are shown for RCP8.5 (pink area) and RCP2.6 (blue area) concentration pathways. Dotted lines show the median temperature in the ensemble projection, while solid colored lines show the evolution of the actual GCM for the corresponding scenario.
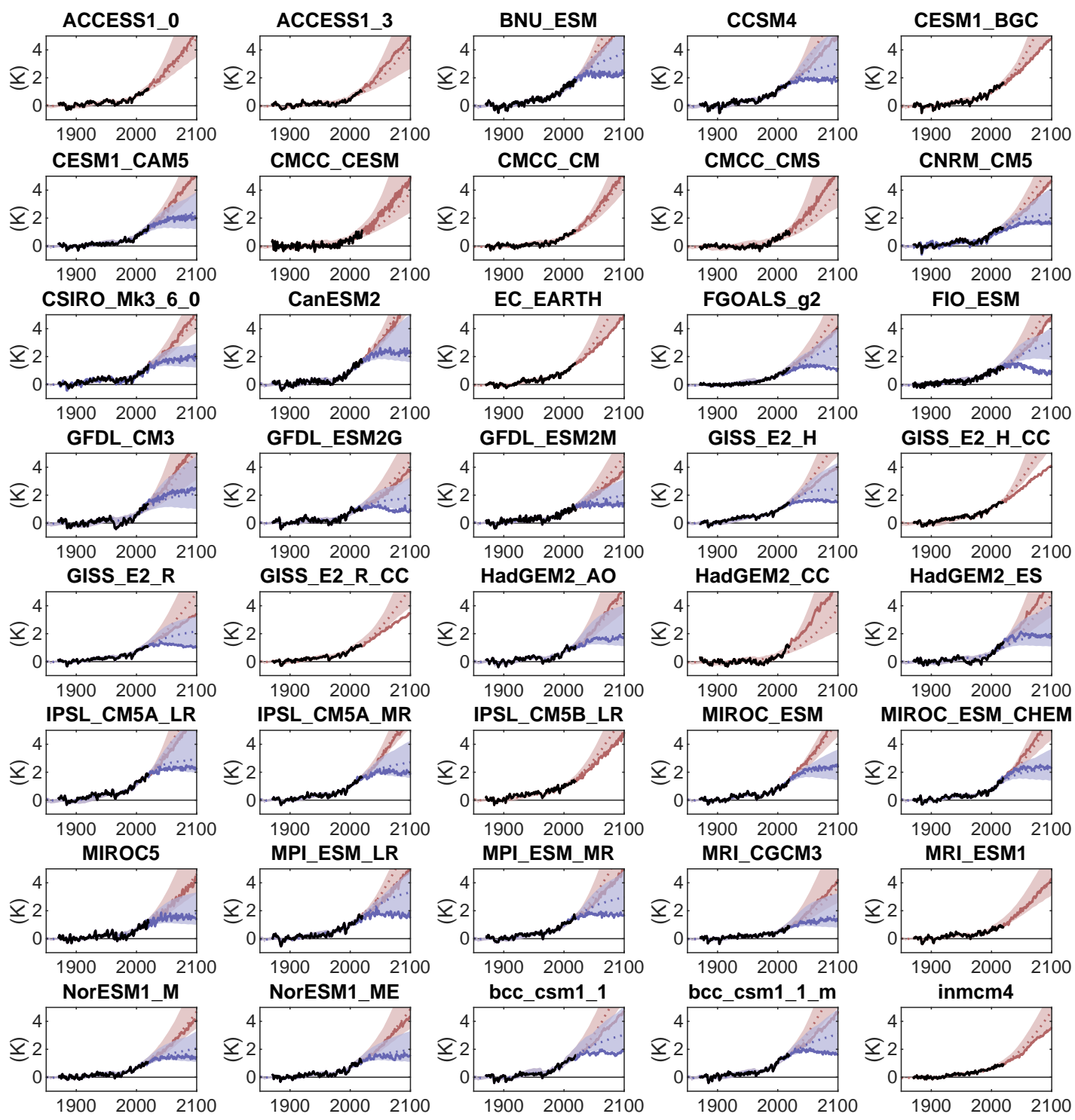
Figure S4. Figure illustrating the 'correction' employed for TCR and ECS in Figure 6. Corrected baseline temperatures are estimated by regression of the first 20 years of the control simulation, and branch-point from the control simulation is identifying by finding the year in which a linear fit to the control model evolution intersects the corrected baseline temperature. Branching in cases where there is no intersection are illustrated by the year in which the trendline is closest to the corrected baseline (either the first or last year).
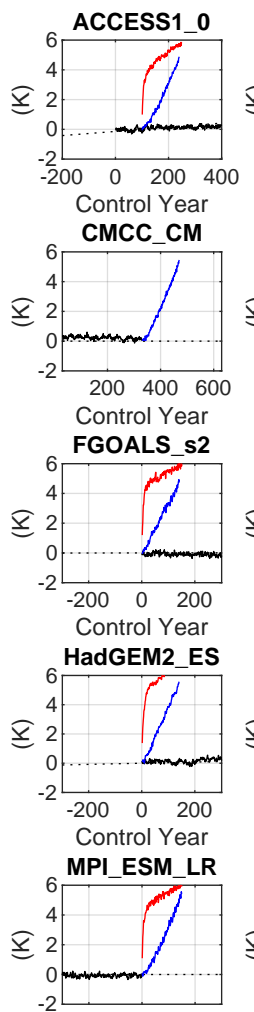

$2 \frac{10001200}{800}$ Control Year

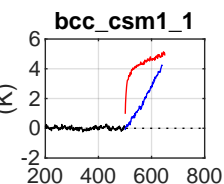

Control Year
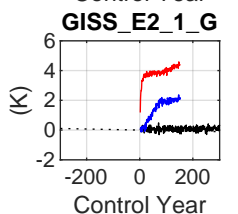
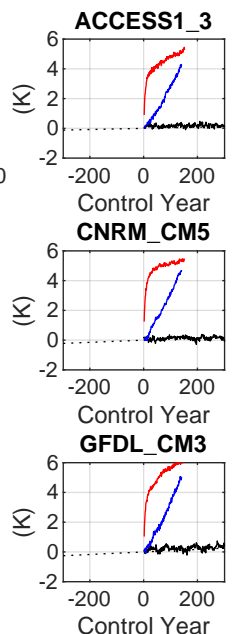

Control Year
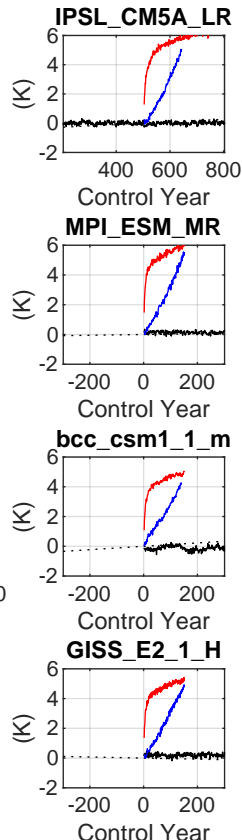
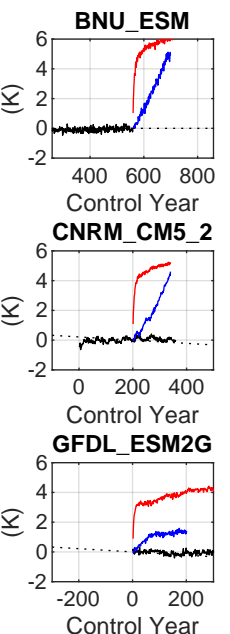

Control Year
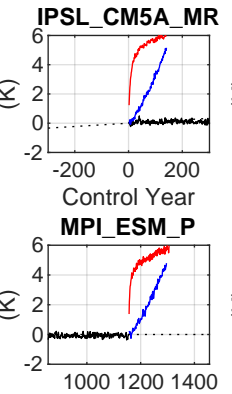

Control Year
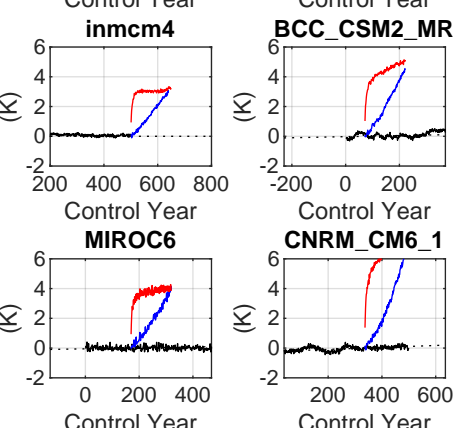
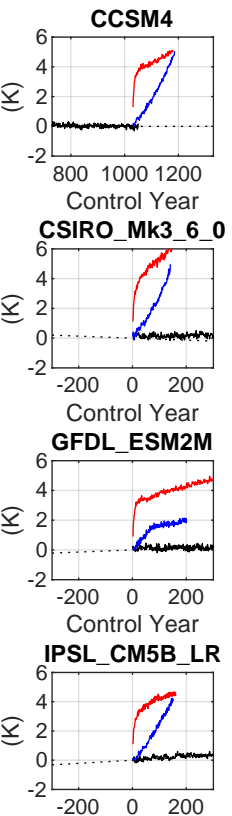

Control Year

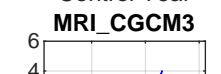

4
$\simeq$

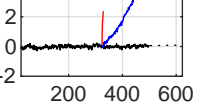

Control Year

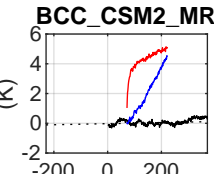

Control Year

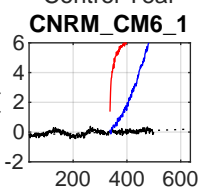

Control Year

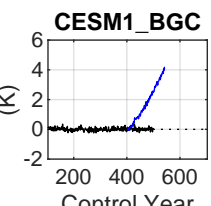

Control Year

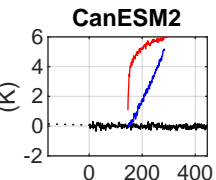

Control Year

GISS E2 H

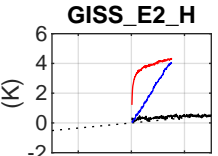

$\begin{array}{lll}-200 & 0 & 200\end{array}$

Control Year
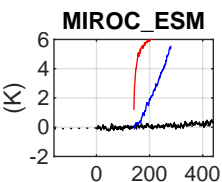

Control Yea

NorESM1 M

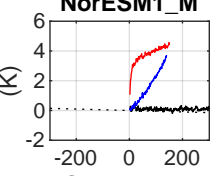

Control Year

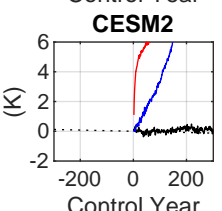

CNRM_ESM2_1

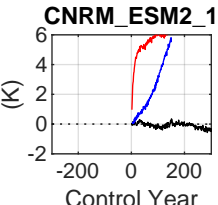

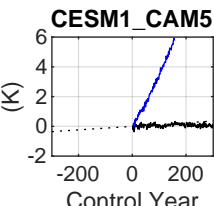
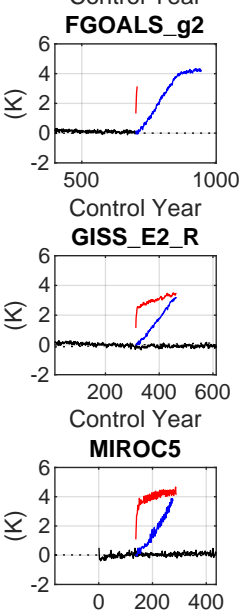

Control Year

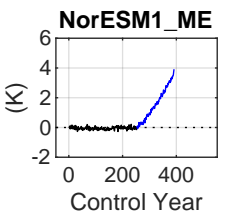

CESM2 WACCM
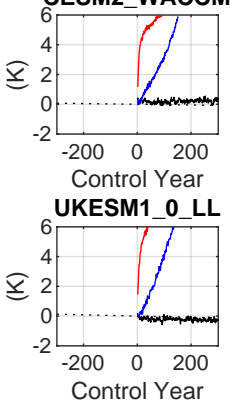
Figure S5. A figure illustrating the log of the sum squared error in best-fitting of the first 140 years of the abrupt $4 \mathrm{xCO} 2$ time series in global mean temperature for each available model in the CMIP5 ensemble, as a function of the number of exponential modes allowed in the pulse response model. Each point shows the error in fitting one model in the CMIP5 ensemble, with colored lines tracking the error in the fitting as a function of number of allowed modes.

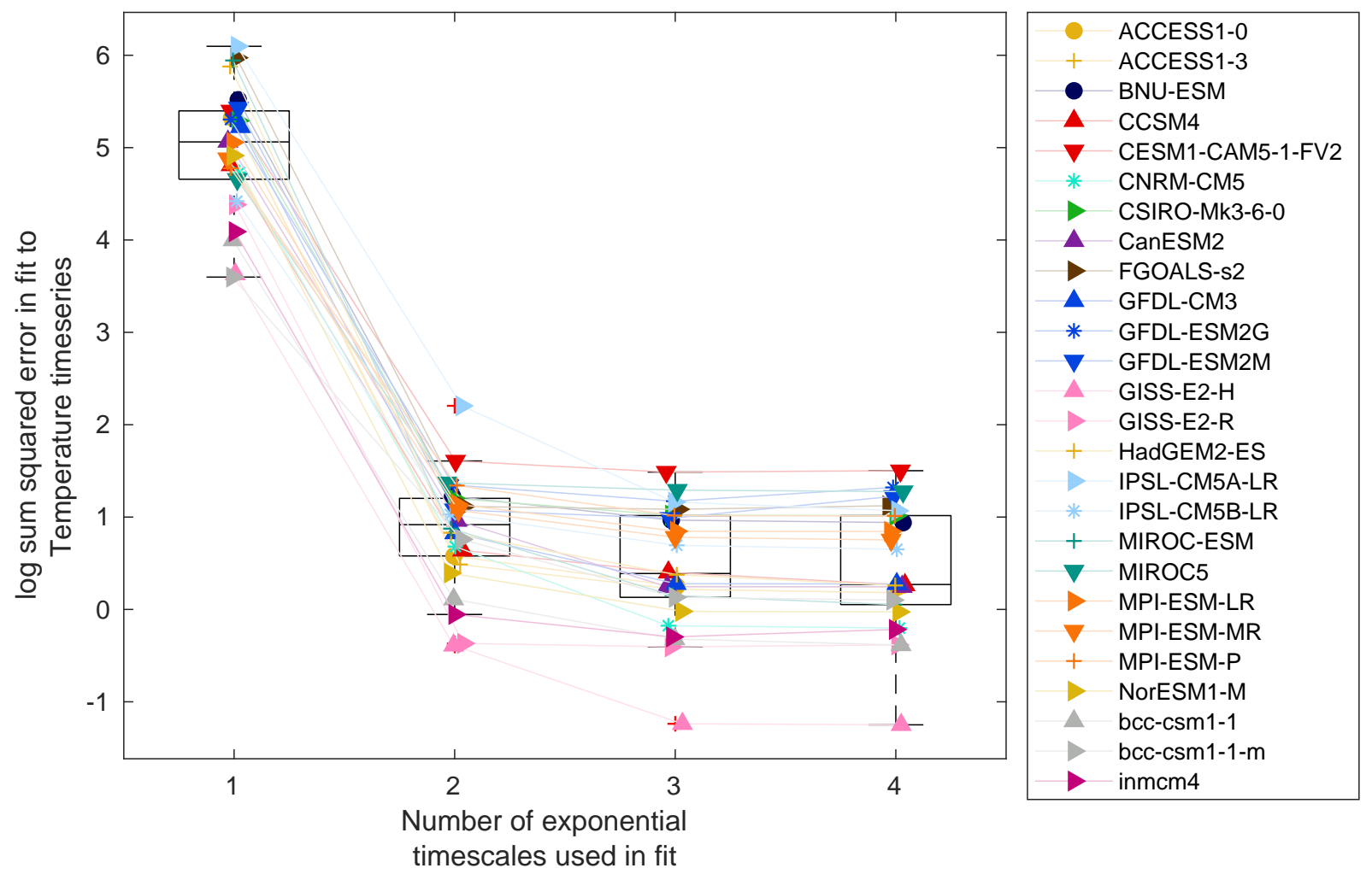

\section{References}

IPCC: Climate Change 2013: The Physical Science Basis. Contribution of Working Group I to the Fifth Assessment Report of the Intergovernmental Panel on Climate Change, Cambridge University Press, Cambridge, United Kingdom and New York, NY, USA, https://doi.org/10.1017/CBO9781107415324, www.climatechange2013.org, 2013. 